\title{
On the Early In Situ Formation of Pluto's Small Satellites
}

\author{
Jason Man Yin Woo ${ }^{1,3}$ and Man Hoi Lee ${ }^{1,2}$ (iD \\ ${ }^{1}$ Department of Earth Sciences, The University of Hong Kong, Pokfulam Road, Hong Kong \\ ${ }^{2}$ Department of Physics, The University of Hong Kong, Pokfulam Road, Hong Kong \\ ${ }^{3}$ Earth-Life Science Institute, Tokyo Institute of Technology, Meguro, Tokyo 152-8550, Japan \\ Received 2017 April 17; revised 2018 February 16; accepted 2018 February 26; published 2018 March 29
}

\begin{abstract}
The formation of Pluto's small satellites-Styx, Nix, Keberos, and Hydra-remains a mystery. Their orbits are nearly circular and are near mean-motion resonances and nearly coplanar with Charon's orbit. One scenario suggests that they all formed close to their current locations from a disk of debris that was ejected from the Charonforming impact before the tidal evolution of Charon. The validity of this scenario is tested by performing $N$-body simulations with the small satellites treated as test particles and Pluto-Charon evolving tidally from an initial orbit at a few Pluto radii with initial eccentricity $e_{\mathrm{C}}=0$ or 0.2 . After tidal evolution, the free eccentricities $e_{\text {free }}$ of the test particles are extracted by applying fast Fourier transformation to the distance between the test particles and the center of mass of the system and compared with the current eccentricities of the four small satellites. The only surviving test particles with $e_{\text {free }}$ matching the eccentricities of the current satellites are those not affected by meanmotion resonances during the tidal evolution in a model with Pluto's effective tidal dissipation function $Q=100$ and an initial $e_{\mathrm{C}}=0.2$ that is damped down rapidly. However, these test particles do not have any preference to be in or near 4:1, 5:1, and 6:1 resonances with Charon. An alternative scenario may be needed to explain the formation of Pluto's small satellites.
\end{abstract}

Key words: celestial mechanics - Kuiper belt objects: individual (Pluto) - planets and satellites: individual (Styx, Nix, Kerberos, Hydra)

\section{Introduction}

Pluto, the dwarf planet visited by the New Horizons spacecraft in 2015 July, has a complex satellite system. With five satellites in total, from closest to furthest, they are Charon, Styx, Nix, Kerberos, and Hydra. Charon, discovered in 1978 (Christy \& Harrington 1978), is the largest one, which has a radius about half of Pluto's and a mass around one-eighth of Pluto's (e.g., Stern et al. 2015). The satellite-to-planet mass ratio is high enough for Pluto and Charon to be regarded as a binary system, as the center of mass of the system is located outside both bodies. Other objects orbiting around the barycenter of the binary are the four much smaller satellites that remained undiscovered until the Hubble Space Telescope imaged them in the early 21 st century (Weaver et al. 2006; Showalter et al. 2011, 2012). Table 1 shows the orbital and physical parameters of the four small satellites.

Astronomers are intrigued to understand the formation of Pluto's satellites due to their special orbital characteristics. All satellites are orbiting on nearly coplanar and nearly circular orbits (Brozović et al. 2015; Showalter \& Hamilton 2015). The orbital period ratios of the four small satellites are close to 1:3:4:5:6 with respect to Charon. Although close to integer ratios, the period ratios are significantly off from integer ratios, and the four small satellites are not trapped in mean-motion resonances (MMR) with Charon (Brozović et al. 2015; Showalter \& Hamilton 2015). Besides, Nix and Hydra are close to a mutual 3:2 MMR (Lee \& Peale 2006; Brozović et al. 2015; Showalter \& Hamilton 2015).

The most widely accepted scenario for the formation of Charon is the intact capture scenario in which Charon was the impactor captured in a giant collision that most likely happened when the population of Kuiper Belt objects was much higher than today (Canup 2005). Charon ended up in an eccentric orbit with semimajor axis about $4 R_{\mathrm{P}}$ (where $R_{\mathrm{P}}=1187 \mathrm{~km}$ is the radius of Pluto) (Canup 2005). Tidal evolution eventually brought them into the double synchronous state-the end state of tidal evolution with the orbital and spin periods of the two bodies being exactly the same and the orbit having zero eccentricity. Pluto and Charon are now $\sim 17 R_{\mathrm{P}}$ from each other.

However, for the small satellites, there is not yet a complete and consistent scenario to explain their formation and how they ended up in their unusual orbits. Several scenarios have been proposed. The first one is known as forced resonant migration, which was proposed by Ward \& Canup (2006). In this scenario, Nix and Hydra (and presumably Styx and Kerberos, which were still undiscovered in 2006) were debris from the giant impact that formed Charon. They formed closer to Pluto than their current locations. When Charon evolved outward due to tidal evolution, the small satellites were caught into corotation resonances with Charon and moved outward together with Charon. The small satellites can be carried to their current positions without instability, because their orbital eccentricities would not be forced up by corotation resonances. When Charon finished tidal evolution, its orbital eccentricity was damped down to zero and the small satellites would escape from the resonances with Charon. However, Lithwick \& Wu (2008) ruled out this idea. They found that in order to transport Nix, Charon's eccentricity $e_{\mathrm{C}}$ should be smaller than 0.024 . Otherwise, overlapping of the second order Lindblad resonance with the corotation resonance at 4:1 would lead to chaos. On the other hand, to transport Hydra, $e_{\mathrm{C}}$ should be larger than 0.04. Otherwise, Hydra would slip out of resonance, because its migration would be faster than libration. Because these two constraints contradict with each other, forced resonant migration in corotation resonance is an unsuccessful scenario.

If transport in corotation resonance does not work, how about transport by capture into multiple resonances at the same mean-motion commensurability? Cheng et al. (2014b) 
Table 1

Orbital and Physical Parameters of the Four Small Satellites of Pluto

\begin{tabular}{|c|c|c|c|c|}
\hline & Styx & Nix & Kerberos & Hydra \\
\hline Semimajor axis $a(\mathrm{~km})$ & 42656 & 48694 & 57783 & 64738 \\
\hline Eccentricity $e\left(10^{-3}\right)$ & 5.787 & 2.036 & 3.280 & 5.862 \\
\hline Period $P$ (days) & 20.16155 & 24.85463 & 32.16756 & 38.20177 \\
\hline$P / P_{\mathrm{C}}$ & 3.156542 & 3.891302 & 5.036233 & 5.980963 \\
\hline$G M\left(10^{-3} \mathrm{~km}^{3} \mathrm{~s}^{-2}\right)$ & $0.0+1.0$ & $3.0 \pm 2.7$ & $1.1 \pm 0.6$ & $3.2 \pm 2.8$ \\
\hline Size $(\mathrm{km})$ & $16 \times 9 \times 8$ & $50 \times 35 \times 33$ & $19 \times 10 \times 9$ & $65 \times 45 \times 25$ \\
\hline Geometric albedo & $0.65 \pm 0.07$ & $0.56 \pm 0.05$ & $0.56 \pm 0.05$ & $0.83 \pm 0.08$ \\
\hline
\end{tabular}

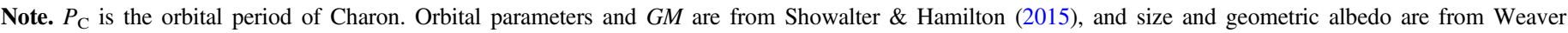
et al. (2016).

investigated this situation and found that although stable capture and transport in 5:1, 6:1, and 7:1 commensurabilities are possible, it is also unlikely for this scenario to work because their results show that the satellites that survive to the end of tidal evolution would have significant final eccentricities. Besides, no stable capture and transport at 3:1 and 4:1 commensurabilities can be observed from their simulations. Because of the expected initial fast rotation of Pluto, a large gravitational coefficient $J_{2}$ of Pluto was also tested. They found that large $J_{2}$ of Pluto causes the resonances at the same commensurability to be further apart from each other and hence libration in multiple resonances simultaneously is much more difficult. This destroys the condition for stable migration in multiple resonances for the small satellites.

Another scenario suggested by Lithwick \& Wu (2008), known as the collisional capture scenario, was studied by Pires dos Santos et al. (2012). In this scenario, a planetesimal orbiting around the Sun could be temporarily captured by the Pluto-Charon binary, which finished tidal evolution. The captured planetesimal collided with another incoming planetesimal and formed a debris disk. Collisions between bodies in the disk damped down the orbital eccentricities and inclinations, leading to the formation of a coplanar and circular debris disk in which the small satellites can be formed near their current positions. However, this scenario was ruled out by Pires dos Santos et al. (2012), as they found that the timescale of temporary capture for objects that are massive enough to produce Nix and Hydra is much smaller than the timescale for another object to come in and collide with it. Their assumed masses for Nix and Hydra are adopted from Tholen et al. (2008). Besides, Walsh \& Levison (2015) investigated how the debris disk would evolve if such a collision did occur and found that the satellites formed would have no strong preference to be in or near resonances. Based on these studies, the collisional capture scenario may not be the answer.

Similar to the collisional capture scenario, Kenyon \& Bromley (2014) suggested that the four small satellites could be formed near their current positions but with a different process. In this early in situ formation scenario, the Charonforming giant impact produced a debris ring at around $20 R_{\mathrm{P}}$. Kenyon \& Bromley (2014) estimated that the transfer of angular momentum from the central binary to the ring could lead to the spreading of the ring to the current positions of the satellites within 5-10 years, which is much faster than the tidal evolution rate of Pluto and Charon. Later, in their second paper, Bromley \& Kenyon (2015) performed a complete study on the spreading of the collisional disk around the tidally evolving Pluto-Charon. They found that the spreading of the ring can occur on a timescale comparable to the formation timescale of the satellites. With such a rapid spreading process, satellites can form near their current positions when Charon was still close to Pluto. Recent crater counting data from New Horizons imply that the surface ages of Nix and Hydra are at least 4 billion years (Weaver et al. 2016). This supports the early formation of the four small satellites. However, the effect of outward tidal evolution of Charon has not been investigated in this scenario. The outwardly evolving Charon would perturb the orbits of the small satellites (for instance, by MMR) and hence they may not be able to lie on nearly circular and coplanar orbits after Charon completed its tidal evolution.

In this paper, we test the plausibility of the early in situ formation scenario by investigating the effects of the tidal evolution of Charon's orbit on the small satellites' mean distances and eccentricities, after the small satellites formed near their current positions from a debris disk. We also examine the final orbits of the satellites in order to see if their orbits are near MMR with Charon. We present our methods and results in Sections 2 and 3, respectively. The results are summarized and discussed in Section 4.

\section{Methods}

We adopt the masses and radii of Pluto and Charon from Brozović et al. (2015): $\quad G M_{\mathrm{P}}=869.61 \mathrm{~km}^{3} \mathrm{~s}^{-2}$, $G M_{\mathrm{C}}=105.88 \quad \mathrm{~km}^{3} \mathrm{~s}^{-2}, \quad R_{\mathrm{P}}=1181 \mathrm{~km}, \quad$ and $R_{\mathrm{C}}=603.6 \mathrm{~km}$, where $M_{\mathrm{P}}, M_{\mathrm{C}}, R_{\mathrm{P}}$, and $R_{\mathrm{C}}$ are the masses and radii of Pluto and Charon and $G$ is the gravitational constant. The gravitational harmonic coefficients $J_{2}$ and $C_{22}$ of Pluto and Charon are set to zero throughout our calculation, where $C_{22}$ is the permanent quadrupole moment. Based on the giant impact hypothesis, Charon is placed at $a_{\mathrm{PC}}=4 R_{\mathrm{P}}$ initially (Canup 2005). The initial orbital eccentricity of Charon, $e_{\mathrm{C}}$, is set to be 0 or 0.2 . The initial $e_{\mathrm{C}}=0.2$ case corresponds to Charon formation according to the intact capture scenario. Zero eccentricity corresponds to Charon forming from a debris disk generated from the giant impact, as Canup (2005) does not rule out this situation although it is less likely. We assume that the initial spin rate of Charon is twice the initial mean motion. Then the initial spin rate of Pluto is $\sim 5.65$ times the initial mean motion, calculated from the current total angular momentum with $a_{\mathrm{PC}}=4 R_{\mathrm{P}}$.

For the small satellites, we treat them as massless test particles and place them randomly in the current orbital distance range of the four small satellites, about $35-60 R_{\mathrm{P}}$ from the barycenter of the binary. To imitate the situation where the satellites formed in a collisional debris disk near their current positions, their initial orbits (coplanar with Charon's orbit) are 
obtained from an integration with eccentricity damping applied to the test particles. We use the Wisdom-Holman (Wisdom \& Holman 1991) integrator in the SWIFT package (Levison \& Duncan 1994), modified for integrations of systems with comparable masses such as Pluto-Charon (Lee \& Peale 2003). We apply eccentricity damping to the test particles as half steps before and after each Wisdom-Holman step (Lee \& Peale 2002), with a damping equation

$$
e_{\text {final }}=e_{\text {initial }} \exp (-0.5 k \delta t),
$$

where $e_{\text {initial }}$ and $e_{\text {final }}$ are the osculating eccentricities before and after damping, respectively, $\delta t \sim 630 \mathrm{~s}$ is the time step we adopted for the damping calculations, and $k=10^{-10} \mathrm{~s}^{-1}$ is the damping coefficient we adopted. Eventually, after about 2000 years, the test particles are damped to nearly the coldest orbits. For test particle orbiting a binary, the coldest orbit is the one with the amplitude of the epicyclic motion, i.e., the free eccentricity $e_{\text {free }}$, equal to zero, as the oscillations forced by the non-axisymmetric components of the binary's potential, including the forced eccentricity

$$
e_{\text {forced }} \approx \frac{5}{4} e_{\mathrm{C}} \frac{M_{\mathrm{P}}-M_{\mathrm{C}}}{M_{\mathrm{P}}+M_{\mathrm{C}}} \frac{a_{\mathrm{PC}}}{R_{0}},
$$

cannot be damped down through collisions between particles within the debris disk (Leung \& Lee 2013). In Equation (2), $a_{\mathrm{PC}}$ is the orbital semimajor axis of Pluto-Charon, and $R_{0}$ is the average distance between the test particle and the center of mass of Pluto-Charon. For $e_{\mathrm{C}}=0, e_{\text {forced }}$ equals to zero according to Equation (2). For $e_{\mathrm{C}}=0.2$ and $a_{\mathrm{PC}}=4 R_{\mathrm{P}}, e_{\text {forced }}$ is around $0.01-0.02$ at the distances of the test particles. The free eccentricity $e_{\text {free }}$ of the test particles at the end of the damping calculation is about 10 times smaller than $e_{\text {forced }}$.

After the eccentricity damping calculation, the whole system is then integrated in the tidal evolution code developed by Cheng et al. (2014a). Two tidal models are used: constant $\Delta t$ and constant $Q$, where $\Delta t$ is the time lag of the tidal bulge of Pluto and $Q$ is the effective tidal dissipation function of Pluto. In the constant $\Delta t$ model, the phase angle of Pluto's tidal bulge $\alpha=\sigma_{l m} \Delta t$ when $\alpha$ is small, where $\sigma_{l m}$ is the tidal frequency, which depends on the mean motion and spin angular velocity of Pluto. Thus, $\alpha$ changes continuously throughout the tidal evolution. In the constant $Q$ model, $\alpha=\operatorname{sgn}\left(\sigma_{l m}\right) / Q$ when $\alpha$ is small, and $\alpha$ is independent of the tidal frequency, except for the sign. Because $\alpha$ has different dependence on tidal frequency, orbital evolution in the constant $Q$ model is qualitatively different from the evolution in the constant $\Delta t$ model, especially near the end of tidal evolution (see Figure 1).

Another major difference between the two models is the evolution timescale. We adopt $\Delta t=600 \mathrm{~s}$ for constant $\Delta t$ and $Q=100$ for constant $Q$, as in Cheng et al. (2014a). Due to the values of $\Delta t$ and $Q$ adopted, constant $Q$ models would take 5-10 times longer to evolve to the doubly synchronous state than the constant $\Delta t$ models. We adopt an initial integration time step of $1000 \mathrm{~s}$. For constant $\Delta t$, due to the expansion of the orbital semimajor axis of Charon, we increase the time step to $5000 \mathrm{~s}$ when Charon evolves to $\sim 10 R_{\mathrm{P}}$ from Pluto, until the end of tidal evolution $\left(\sim 10^{6}\right.$ years). For constant $Q$, we adopt a similar procedure, except for an extra increase of the time step to $10000 \mathrm{~s}$ at $\sim 3.17 \times 10^{6}$ years after the start of the simulation, due to the longer tidal evolution time ( $\sim 10^{7}$ years). The readers are referred to Cheng et al. (2014a) for details of the tidal models and the reasons for adopting various parameter values for the tidal models and the numerical integrations.

Two values for the relative rate of tidal dissipation in Charon and Pluto, $A$, are adopted for each model. For constant $\Delta t$,

$$
A=\frac{k_{2 \mathrm{C}}}{k_{2 \mathrm{P}}} \frac{\Delta t_{\mathrm{C}}}{\Delta t}\left(\frac{M_{\mathrm{P}}}{M_{\mathrm{C}}}\right)^{2}\left(\frac{R_{\mathrm{C}}}{R_{\mathrm{P}}}\right)^{5},
$$

where $k_{2 \mathrm{C}}$ and $k_{2 \mathrm{P}}$ are the Love numbers of Charon and Pluto, respectively, and $\Delta t_{\mathrm{C}}$ is the time lag of Charon's tidal bulge (Mignard 1980; Cheng et al. 2014a). For constant $Q$,

$$
A=\frac{k_{2 \mathrm{C}}}{k_{2 \mathrm{P}}} \frac{Q}{Q_{\mathrm{C}}}\left(\frac{M_{\mathrm{P}}}{M_{\mathrm{C}}}\right)^{2}\left(\frac{R_{\mathrm{C}}}{R_{\mathrm{P}}}\right)^{5},
$$

where $Q_{\mathrm{C}}$ is the effective tidal dissipation function of Charon (Yoder \& Peale 1981; Cheng et al. 2014a). We adopt $A=10$ or 40 for constant $\Delta t$ and $A=0.65$ or 2.5 for constant $Q$. Figure 1 shows the evolution of Charon's eccentricity throughout the tidal evolution in the constant $\Delta t$ and constant $Q$ models, with initial $e_{\mathrm{C}}=0.2$. For the smaller $A$ values ( $A=10$ for constant $\Delta t$ and $A=0.65$ for constant $Q$ ), $e_{\mathrm{C}}$ stays around 0.2 until near the end of tidal evolution. For the larger $A$ values $(A=40$ for constant $\Delta t$ and $A=2.5$ for constant $Q), e_{\mathrm{C}}$ damps down rapidly to near zero when $t \sim 10^{3}$ years for constant $\Delta t$ and $t \sim 10^{5}$ years for constant $Q$. Therefore, the damping rate of $e_{\mathrm{C}}$ depends on the value of $A$.

We integrate 200 test particles with different initial semimajor axes and eccentricities (before the eccentricity damping calculation) for each combination of $A$ and initial $e_{\mathrm{C}}$. We should emphasize that we are not modeling a debris disk but a set of individual satellites that have already formed within a debris disk. Table 2 shows the combination of $A$ and $e_{\mathrm{C}}$ we integrate in each tidal model. For initial $e_{\mathrm{C}}=0$, we only integrate with larger $A$, as $e_{\mathrm{C}}$ would stay at zero throughout the tidal evolution and different $A$ values would not affect the evolution of $e_{\mathrm{C}}$.

After the tidal evolution of Charon, the surviving test particles are then integrated for another 800 days and we apply fast Fourier transformation (FFT) to the distance $R(t)$ between the test particle and the center of mass of the system to calculate the magnitude of the free eccentricity, $e_{\text {free}}$, from the power in the peak at the epicyclic frequency $\kappa_{0}$ in the power spectrum (see Woo \& Lee 2018 for details). The reason for extracting $e_{\text {free }}$ of the test particles from the power spectrum obtained from FFT is that the osculating Keplerian eccentricity $e_{\text {osc }}$ can show significant variations due to the oscillations forced by the Pluto-Charon binary and that even the mean of $e_{\text {osc }}$ can be significantly different from $e_{\text {free }}$. In addition, $e_{\text {free }}$ of the current satellites are closer to the eccentricities obtained by Showalter \& Hamilton (2015) from fitting the orbits of the small satellites by precessing ellipses. Figure 2 shows the orbital integration of two of the small satellites, Nix and Kerberos, using the best-fit data of Showalter \& Hamilton (2015). We observe that there are obvious differences between $e_{\text {osc }}$ and $e_{\text {free }}$ for each satellite, with the mean of $e_{\mathrm{osc}}$ at least a factor of three larger than $e_{\text {free}}$, and that $e_{\text {free }}$ is much closer to the fitting result of Showalter \& Hamilton (2015) listed in Table 1. Therefore, we decide to compare $e_{\text {free }}$ of the test particles with the eccentricities of the small satellites listed in Table 1. 

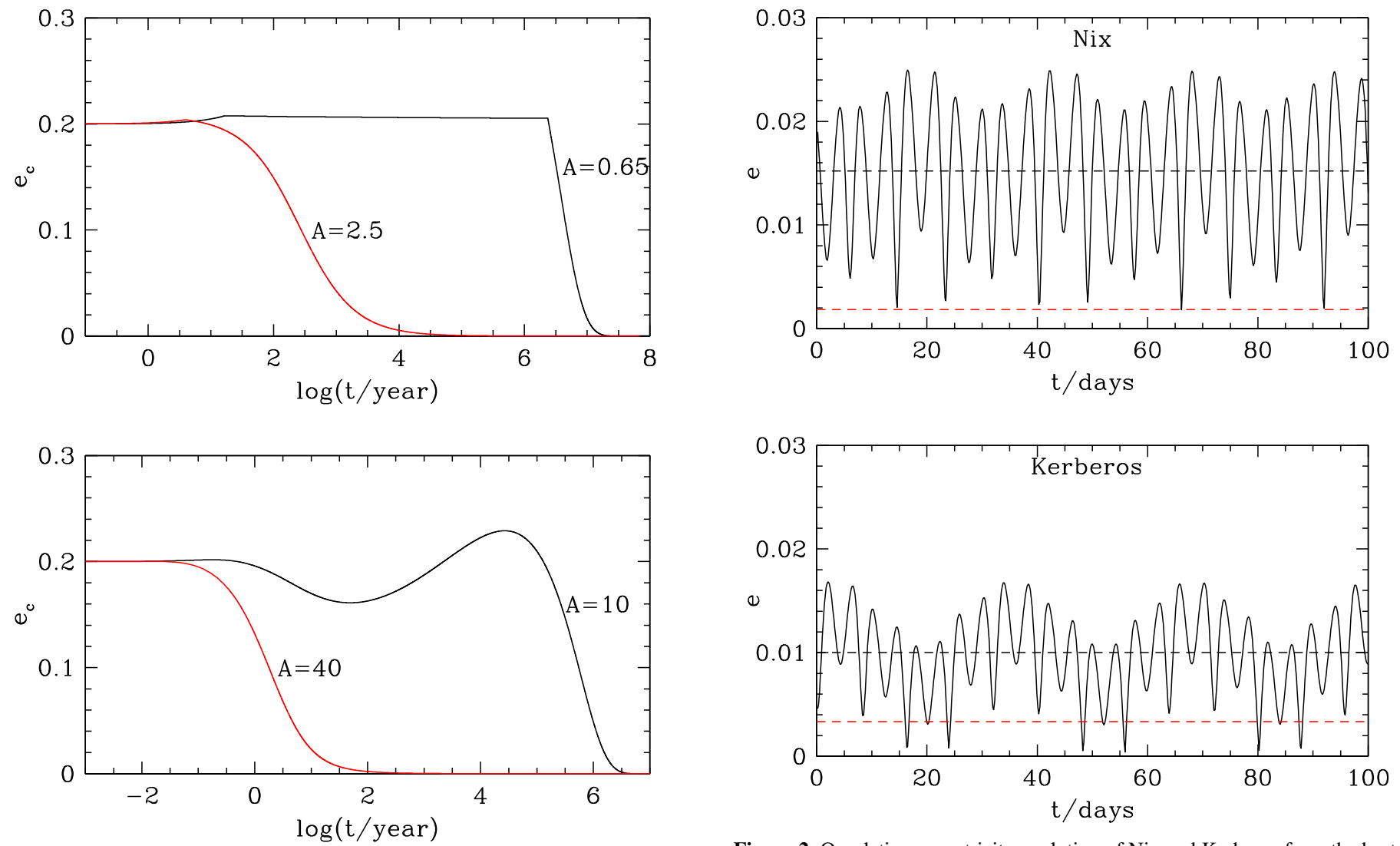

Figure 1. Tidal evolution of the orbital eccentricity of Charon, $e_{\mathrm{C}}$. The upper panel shows the constant $Q$ model with $Q=100$, and the lower panel shows the constant $\Delta t$ model with $\Delta t=600 \mathrm{~s}$. In each panel, the black line is the integration with smaller $A$ value, and the red line is the integration with larger $A$ value.

Table 2

Combination of $A$ and Initial $e_{\mathrm{C}}$ in Different Tidal Models

\begin{tabular}{ll}
\hline \hline Constant $\Delta t$ & Constant $Q$ \\
\hline$e_{\mathrm{C}}=0, A=40$ & $e_{\mathrm{C}}=0, A=2.5$ \\
$e_{\mathrm{C}}=0.2, A=10$ & $e_{\mathrm{C}}=0.2, A=0.65$ \\
$e_{\mathrm{C}}=0.2, A=40$ & $e_{\mathrm{C}}=0.2, A=2.5$ \\
\hline
\end{tabular}

The adopted initial integration time step of $1000 \mathrm{~s}$ is more than 60 steps per orbit of Pluto-Charon for initial $a_{\mathrm{PC}}=4 R_{\mathrm{P}}$ and resolves the dominant forced oscillations in the motion of the test particles. We repeat one set of simulations (constant $\Delta t$ with $A=10$ and initial $e_{\mathrm{C}}=0.2$ ) with half and twice the adopted time step. We find that the statistics are identical to those in Table 3 within uncertainties, which confirm that the adopted time step is small enough.

\section{Results}

We first define the resonant terms in the disturbing function in order to explain the resonant behaviors of the test particles. For coplanar orbits, the lowest-order resonant terms at the $m+1: 1$ mean-motion commensurability exterior to Charon are

$$
\Phi_{m}=\frac{G M_{\mathrm{C}}}{a} \sum_{n=0}^{m} f_{m, n}(\alpha) e^{m-n} e_{\mathrm{C}}^{n} \cos \phi_{m, n},
$$

where $\phi_{m, n}=(m+1) \lambda-\lambda_{\mathrm{C}}-(m-n) \varpi-n \varpi_{\mathrm{C}}$ are the resonant angles, $a, e, \lambda$, and $\varpi$ are the orbital semimajor axis,

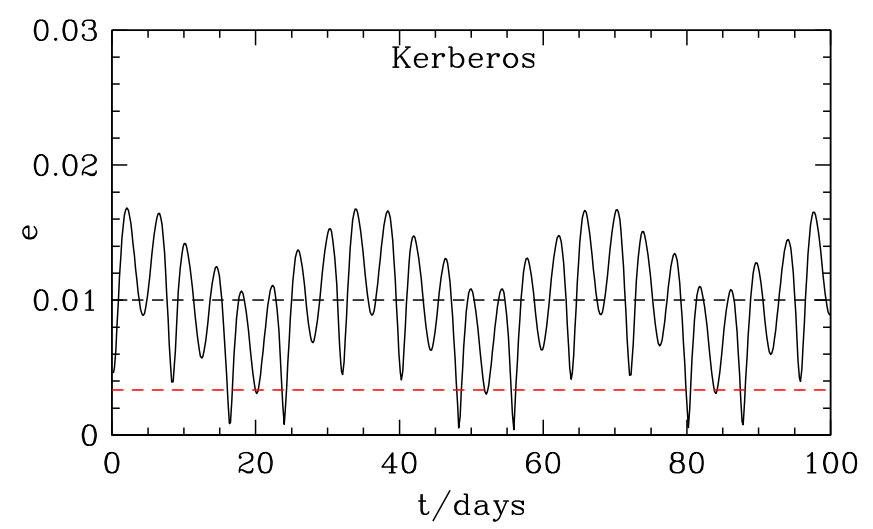

Figure 2. Osculating eccentricity evolution of Nix and Kerberos from the bestfit data of Showalter \& Hamilton (2015). The black solid and dashed lines are the osculating eccentricity $e_{\mathrm{osc}}$ and the mean of $e_{\mathrm{osc}}$, respectively. The red dashed lines are the free eccentricities $e_{\text {free }}$ obtained from FFT.

Table 3

Statistics of Test Particles with Different Evolution

\begin{tabular}{lccccc}
\hline \hline Ejected & $\begin{array}{c}\text { Still } \\
\text { Trapped } \\
\text { in MMR }\end{array}$ & $\begin{array}{c}\text { Once } \\
\text { Trapped } \\
\text { in MMR }\end{array}$ & $\begin{array}{r}\text { Affected } \\
\text { by MMR }\end{array}$ & $\begin{array}{c}\text { Unaffected } \\
\text { by MMR }\end{array}$ \\
\hline \multicolumn{7}{c}{ Small $A$ and Initial $e_{\mathrm{C}}=0.2$} \\
\hline Constant $\Delta t$ & 160 & 4 & 6 & 30 & 0 \\
Constant $Q$ & 190 & 1 & 4 & 5 & 0 \\
\hline \multicolumn{7}{c}{ Large $A$ and Initial $e_{\mathrm{C}}=0.2$} \\
\hline Constant $\Delta t$ & 56 & 47 & 0 & 1 & 96 \\
Constant $Q$ & 41 & 16 & 1 & 16 & 126 \\
\hline \multicolumn{7}{c}{ Large $A$ and Initial $e_{\mathrm{C}}=0$} \\
\hline Constant $\Delta t$ & 0 & 2 & 0 & 2 & 196 \\
Constant $Q$ & 0 & 2 & 0 & 2 & 196 \\
\hline
\end{tabular}

Note. See Section 3 for the definition of each type of evolution.

eccentricity, mean longitude, and longitude of periapse of the small satellite, and the orbital elements with subscript $\mathrm{C}$ are those of Charon (Murray \& Dermott 1999). $f_{m, n}$ are functions of $\alpha=a_{\mathrm{C}} / a$, the Laplace coefficients and their derivatives with respect to $\alpha$.

We now present the results of our simulations. Some of the test particles are ejected during the tidal evolution due to trapping in MMR with Charon. Their eccentricities are forced up to extremely high values. The evolution of the test particles 

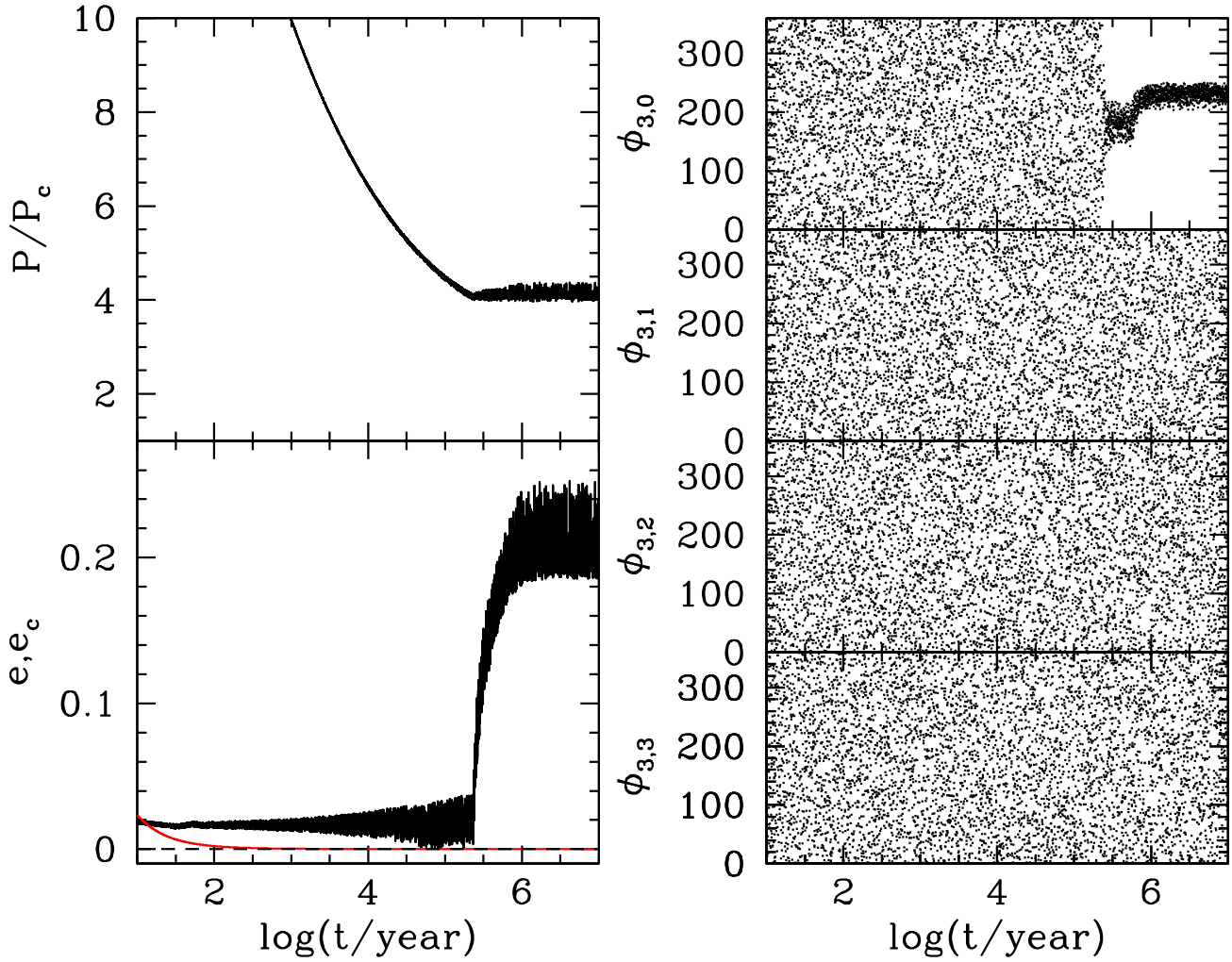

Figure 3. Evolution of the period ratio with respect to Charon (upper left panel), $e_{\mathrm{osc}}$ (lower left panel), and the four resonant angles $\phi_{3, n}=4 \lambda-\lambda_{\mathrm{C}}-(3-n) \varpi-n \varpi_{\mathrm{C}}$ of the $4: 1$ resonance (right panels) for a test particle that is still trapped in resonance at the end of tidal evolution, in the constant $\Delta t$ model with $A=40$ and initial $e_{\mathrm{C}}=0.2$. The red line in the lower left panel shows the evolution of $e_{\mathrm{C}}$ (see Figure 1 for the full evolution of $e_{\mathrm{C}}$ ).

that survive to the end of the tidal evolution falls into four categories :

1. Some test particles are still trapped in MMR with Charon at the end of the tidal evolution (labeled as "Still trapped in MMR" in Table 3). Figure 3 shows a test particle that is trapped in 4:1 MMR with Charon, in the constant $\Delta t$ model with $A=40$ and initial $e_{\mathrm{C}}=0.2$. One of the resonant angles $\phi_{3,0}=4 \lambda-\lambda_{\mathrm{C}}-3 \varpi$ is librating around $\sim 220^{\circ}$ at the end of tidal evolution. The reason is that $e_{\mathrm{C}}$ is damped down to zero when the test particle is trapped into 4:1 MMR. Hence, only the resonant term in $\Phi_{3}$ with $\phi_{3,0}$, which does not depends on $e_{\mathrm{C}}$, is effective at the $4: 1$ commensurability (see Equation (5)). Also, $P / P_{\mathrm{C}}$ ends near 4 and the eccentricity of the test particle is forced up to $\sim 0.2$, which is too large compared to the current eccentricities of the small satellites. Test particles in this category are shown as blue points in Figures 7, 8, and 10 .

2. Some test particles are once trapped in MMR but then escape from the resonance when Charon finishes its tidal evolution (labeled as "Once trapped in MMR" in Table 3). Figure 4 is an example of a test particle that is once trapped in the 7:1 MMR with Charon and then escapes from it, in the constant $\Delta t$ model with $A=10$ and initial $e_{\mathrm{C}}=0.2$. We observe that between $\sim 10^{4}$ and $\sim 10^{6}$ years all seven resonant angles, $\phi_{6, n}=7 \lambda-\lambda_{\mathrm{C}}-(6-n) \varpi-n \varpi_{\mathrm{C}}$ where $n$ are integers from 0 to 6 , are librating. Although $P / P_{\mathrm{C}}$ ends near 7 , all resonant angles are no longer librating but are circulating after $10^{6}$ years, which indicates that the test particle escapes from the 7:1 MMR before the end of the tidal evolution. One of the possible reasons for the escape from MMR is the decreasing $e_{\mathrm{C}}$ from $\sim 10^{5}$ years to the end of the tidal evolution. This would weaken all of the resonant terms in $\Phi_{6}$ (except the one with $\phi_{6,0}$ ). Test particles in this category are shown as red points in Figures 7 and 8 .

3. Some test particles are perturbed but not trapped in MMR, and the eccentricities of the test particles are significantly affected (labeled as "Affected by MMR" in Table 3). Figure 5 is an example of a test particle that is affected by the 4:1 MMR with Charon when it passes through that resonance, in the constant $Q$ model with $A=2.5$ and initial $e_{\mathrm{C}}=0.2$. The osculating eccentricity $e_{\text {osc }}$ of the test particle is forced up to $\sim 0.08$ when the test particle is passing through the 4:1 commensurability at $\sim 10^{6}$ years but none of the resonant angles, $\phi_{3, n}=4 \lambda-\lambda_{\mathrm{C}}-(3-n) \varpi-n \varpi_{\mathrm{C}}$ where $n$ are integers from 0 to 3 , librate. Although the test particle tries to get into $\phi_{3,0}$ in between $10^{5}$ and $10^{6}$ years, but $\phi_{3,0}$ fails to librate. This shows that the test particle is not trapped into the 4:1 MMR with Charon. Test particles in this category are shown as black points in Figures 7, 8, and 10 .

4. Some test particles are not affected by any MMR when they pass through them (labeled as "Unaffected by MMR" in Table 3). Figure 6 demonstrates a case where the test particle is not affected by MMR, in the constant $Q$ model with $A=2.5$ and initial $e_{\mathrm{C}}=0$. The test particle's final $e_{\text {osc }}$ remains at a relatively low value $(\sim 0.02)$ and the final period ratio is not near any integer value. The increase in fluctuations of $e_{\mathrm{osc}}$ and the osculating semimajor axis $a_{\text {osc }}$ is due to the orbit of the test particle 

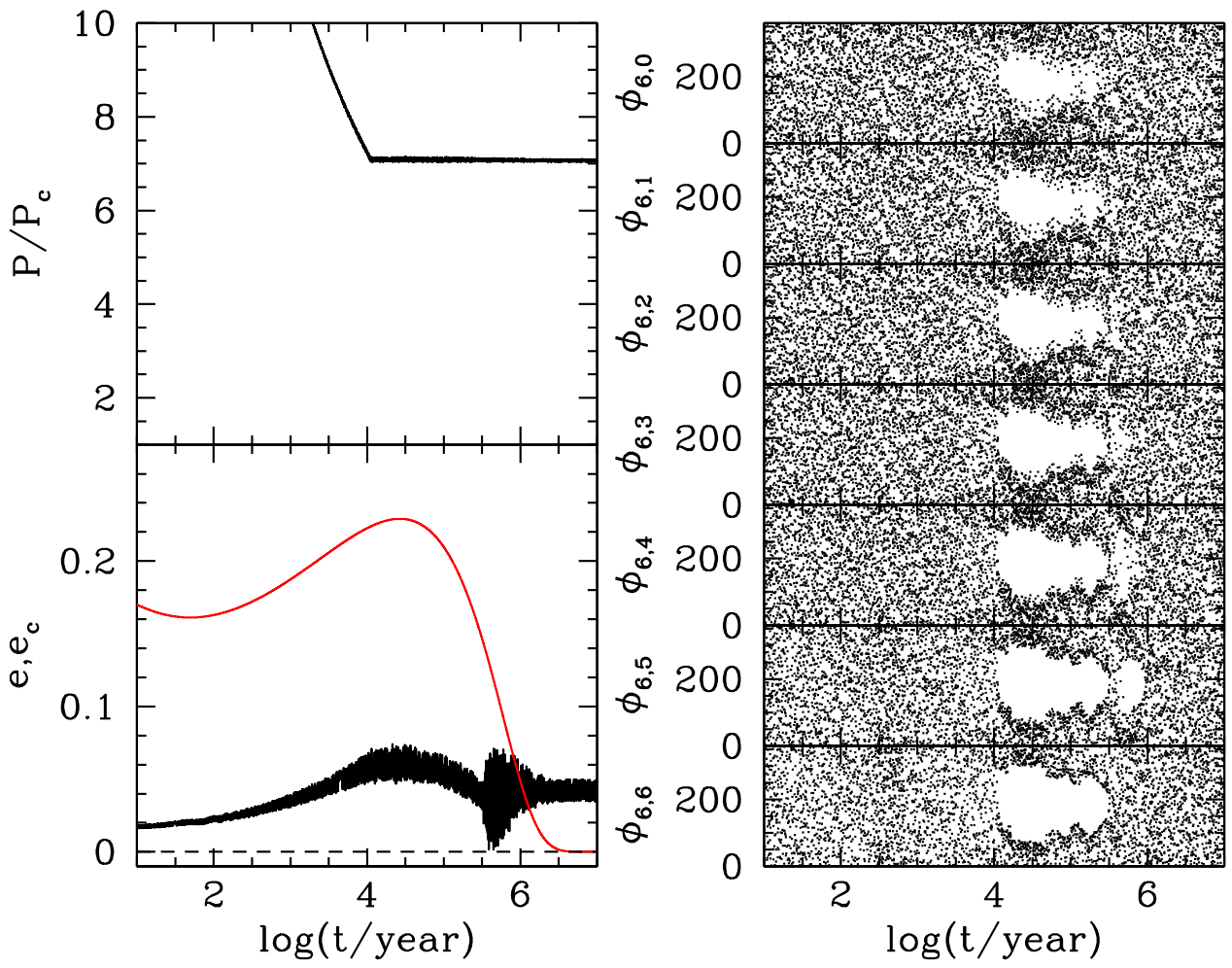

Figure 4. Evolution of the period ratio with respect to Charon (upper left panel), $e_{\text {osc }}$ (lower left panel), and the seven resonant angles $\phi_{6, n}=7 \lambda-\lambda_{\mathrm{C}}-(6-n) \varpi-n \varpi_{\mathrm{C}}$ of the 7:1 resonance (right panels) for a test particle that is trapped but then escape from resonance, in the constant $\Delta t$ model with $A=10$ and initial $e_{\mathrm{C}}=0.2$. The red line in the lower left panel shows the evolution of $e_{\mathrm{C}}$.
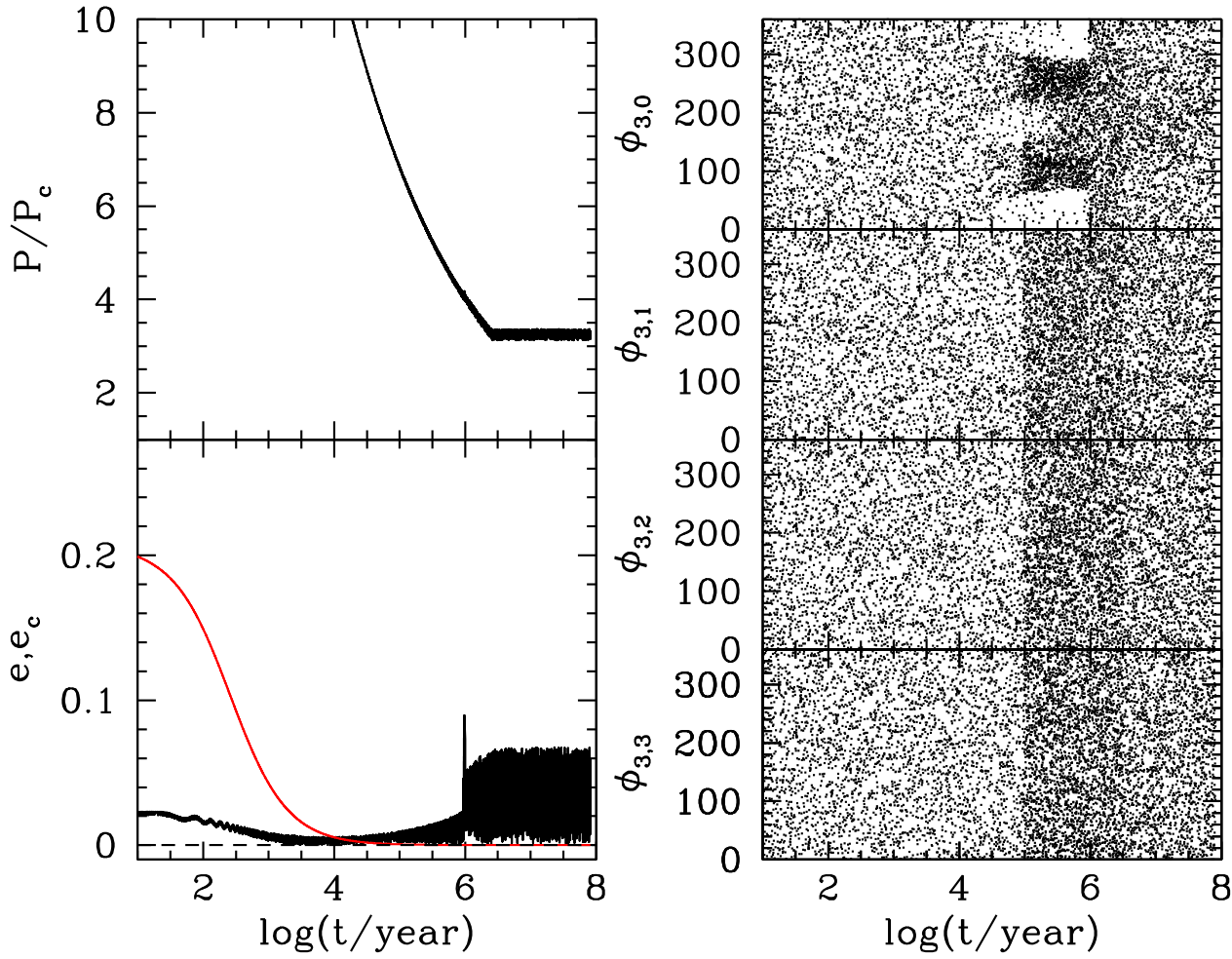

Figure 5. Same as Figure 3, but for a test particle that passes through the 4:1 MMR and is affected but not trapped in the 4:1 MMR, in the constant $Q$ model with $A=2.5$ and initial $e_{\mathrm{C}}=0.2$. The change in density of points in the right panels after $t=10^{5}$ years is due to a change in data sampling frequency.

becoming less Keplerian. The tidal expansion of the orbit of Charon increases the effects of the forced oscillation terms. Test particles in this category are shown as green points in Figures 8 and 10.
Table 3 shows the statistics of the test particles for the six models, which can be grouped into three categories (see Table 2 for the combinations of $A$ and initial $e_{\mathrm{C}}$ we adopted): (1) small $A$ and initial $e_{\mathrm{C}}=0.2$, where $A=10$ for constant $\Delta t$ 

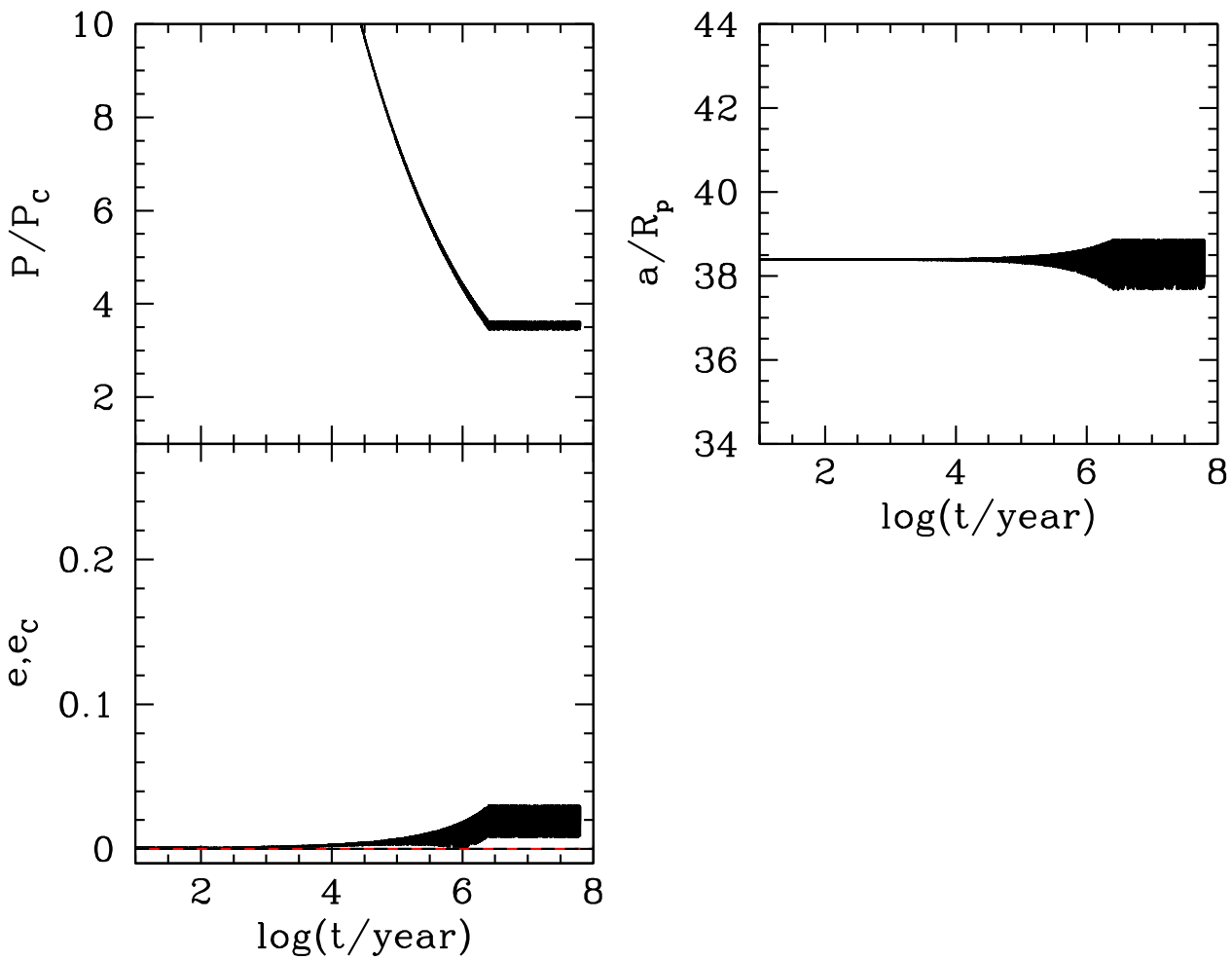

. Evolution of the period ratio with respect to Charon (upper left panel), $e_{\text {osc }}$ (lower left panel), and the osculating semimajor axis $a_{\text {osc }}$ (upper right panel) for a which stays at zero throughout the whole tidal evolution.

and $A=0.65$ for constant $Q ;(2)$ large $A$ and initial $e_{\mathrm{C}}=0.2$, where $A=40$ for constant $\Delta t$ and $A=2.5$ for constant $Q$; and (3) large $A$ and initial $e_{\mathrm{C}}=0$, where $A=40$ for constant $\Delta t$ and $A=2.5$ for constant $Q$.

\subsection{Small $A$ and Initial $e_{C}=0.2$}

Of the $2 \times 200$ test particles (we integrate the same set of 200 test particles in the constant $\Delta t$ and constant $Q$ models), most of them are ejected by the tidally evolving Charon (see Table 3). Because the orbit of Charon stays at high eccentricity until near the end of tidal evolution (see Figure 1), the highorder resonant terms in the disturbing function (e.g., $\Phi_{5}$ or $\Phi_{6}$ in Equation (5)) are strong enough to allow most of the test particles to be trapped into a high-order MMR with Charon (e.g., 6:1 and 7:1) in the early stages of the tidal evolution. This would force up the eccentricities of test particles (Ward \& Canup 2006) and the test particles are ejected from their orbits eventually.

For the remaining $\sim 12 \%$ of the test particles that survive to the end of tidal evolution, all are affected by resonances. This is again due to the large $e_{\mathrm{C}}$ causing the high-order resonant terms in the disturbing function to be strong. Among the surviving test particles, most of them $(\sim 70 \%)$ are affected by passing through resonances but are not trapped in resonances. Only $\sim 20 \%$ are once trapped in resonances but then escape from resonances, and $\sim 10 \%$ are still trapped in resonances at the end of tidal evolution. There are some differences between the results for constant $\Delta t$ and constant $Q$. For example, $\sim 95 \%$ of the test particles in the constant $Q$ model are ejected, compared to only $\sim 80 \%$ in the constant $\Delta t$ model. Also, among the surviving test particles in the constant $\Delta t$ model, $75 \%$ are only affected but not trapped in MMR, whereas only $50 \%$ of the surviving test particles in the constant $Q$ model are in this category. The difference in resonance behavior between constant $\Delta t$ and constant $Q$ could be due to the difference in tidal evolution timescale. Because Charon evolves much slower in the constant $Q$ model, it would be more probable for the test particles to be trapped in MMR with Charon in constant $Q$ than in constant $\Delta t$.

Figure 7 shows the logarithm of $e_{\text {free }}$ of the surviving test particles against their final mean distance to the center of mass of the Pluto-Charon binary, $R_{0}$. Compared to the current four satellites (magenta capital letters in Figure 7), we find some test particles with orbits close to those of Kerberos and Hydra ("K" and " $H$ " in Figure 7). However, nothing matches the orbits of Nix and Styx, as all surviving test particles are located further than the 5:1 MMR with Charon, but Nix and Styx are located closer than the 4:1 MMR. Hence, this scenario is unable to explain the formation of Styx and Nix.

As the number of surviving test particles (40 for constant $\Delta t$ and 10 for constant $Q$ ) is quite small in our simulations with 200 test particles each, there is a possibility that we have missed some survivors that match the orbits of Nix and Styx with a reasonable probability among the survivors. However, we can rule this possibility out for two reasons. First, we perform an additional set of 200 test particles for each model and the statistics are consistent with those listed in Table 3 and shown in Figure 7, with no surviving test particles located closer than the 5:1 MMR. Second, as pointed out by Smullen \& Kratter (2017), the lack of surviving test particles closer than the 5:1 MMR can be explained by applying the instability boundary of Holman \& Wiegert (1999) for circumbinary orbits to the tidally evolving Pluto-Charon binary. They found that the instability boundary evolves beyond the orbits of Styx and 


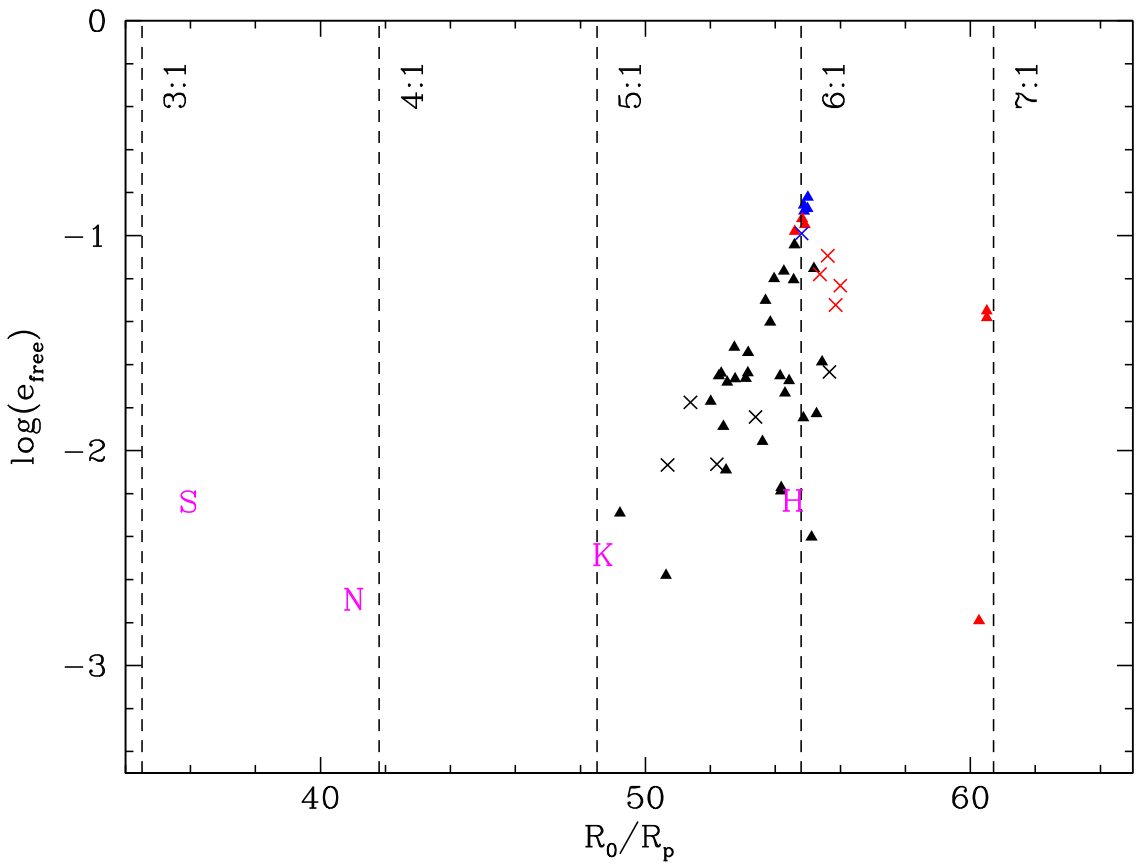

Figure 7. Plot of final $\log \left(e_{\text {free }}\right)$ against final mean distance to the center of mass of the Pluto-Charon binary, $R_{0}$, of the surviving test particles in the small $A$ and initial $e_{\mathrm{C}}=0.2$ integrations. The blue points are test particles that are still trapped in resonance at the end of tidal evolution; the red points are test particles that are trapped but then escape from resonance; and the black points are the test particles that pass through and are affected by resonances but are not trapped. The triangles and crosses correspond to the constant $\Delta t$ and constant $Q$ models, respectively. The four magenta letters indicate $a$ and $\log (e)$ of the current four satellites from Showalter \& Hamilton (2015) (see Table 1). The current 3:1 to 7:1 mean-motion commensurabilities with Charon are shown by the vertical dashed lines

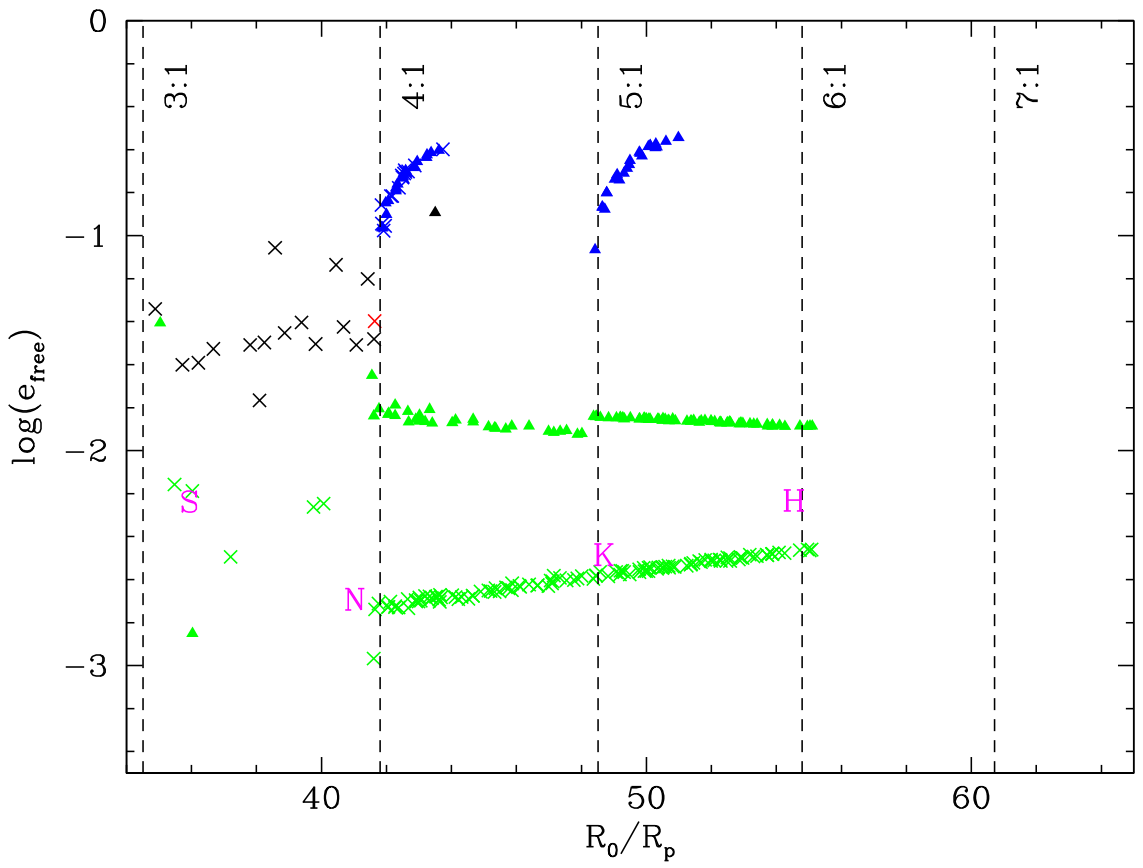

Figure 8. Same as Figure 7, but for the large $A$ and initial $e_{\mathrm{C}}=0.2$ integrations. The green points are test particles that are not affected by resonances.

Nix before it shrinks back to just inside the orbit of Styx, if $e_{\mathrm{C}}$ remains high until near the end of tidal evolution.

\subsection{Large $A$ and Initial $e_{C}=0.2$}

Compared to the small $A$ case (where $\sim 88 \%$ of the test particles are ejected), far fewer test particles $(\sim 24 \%)$ are ejected (see Table 3 ). This is because $e_{\mathrm{C}}$ damps down to 0 very quickly when $A$ is large (see Figure 1). When the regions of high-order resonances like $6: 1$ and 7:1 start to pass through the orbits of the test particles, $e_{\mathrm{C}}$ already damps down to nearly 0 . Only terms with $n=0$ in Equation (5) are effective and the strength of each term mainly depends on the order of the resonance, $m$. The resonant term is stronger if $m$ is smaller. Hence, the test particles are less likely to be trapped in highorder resonances and have a higher chance of survival, unless some lower order resonances (e.g., 4:1 or 5:1 resonance) sweep through their orbits. For the same reason, most of the surviving test particles $(\sim 73 \%)$ are not affected by resonances. 


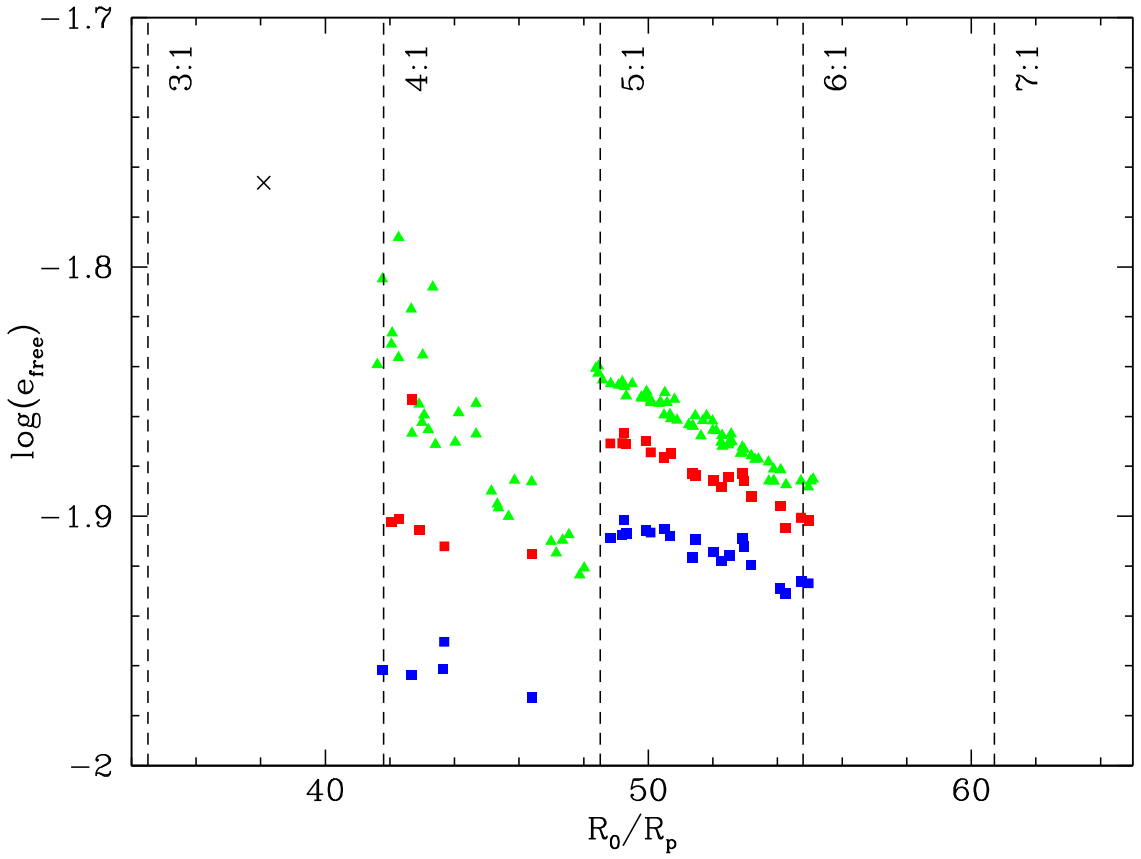

Figure 9. Same as Figure 8, but also with test particles that survive in the integrations with $\Delta t=300 \mathrm{~s}$ (red squares) and $150 \mathrm{~s}$ (blue squares).

Figure 8 shows the plot of final $\log \left(e_{\text {free }}\right)$ against final $R_{0}$ for the test particles that survive to the end of tidal evolution for large $A$ and initial $e_{\mathrm{C}}=0.2$. We observe that the blue points ( $\sim 21 \%$ of the surviving test particles), which represent test particles that are still trapped in MMR with Charon at the end of tidal evolution, are only located close to 4:1 and 5:1 MMR. None are found near the $3: 1,6: 1$ or $7: 1 \mathrm{MMR}$. On the other hand, most of the test particles that pass through and are affected by resonances (black points in Figure 8, which are $\sim 6 \%$ of the surviving test particles) end in between the $3: 1$ and 4:1 MMR.

For the test particles not affected by resonances (green points in Figure 8), we find that the results are different for the constant $\Delta t$ and constant $Q$ models. For constant $\Delta t$, most of the test particles have $e_{\text {free }}$ (green triangles in Figure 8) within the range of $0.01-0.03$, whereas most of the test particles for constant $Q$ have $e_{\text {free }}$ (green crosses in Figure 8) within the range of $10^{-3}-4 \times 10^{-3}$, which is an order of magnitude lower than those for constant $\Delta t$. We also observe that in between the 4:1 and 6:1 MMR, the green crosses form a line with a positive slope (i.e., $e_{\text {free }}$ is larger when the test particle is located further from Pluto-Charon), whereas the green triangles almost form a line with a negative slope (i.e., $e_{\text {free }}$ is smaller when the test particle is located further from Pluto-Charon), except for a jump at the 5:1 MMR.

Compared to the eccentricities of the current satellites, we discover that some of the test particles in the constant $Q$ model that are not affected by resonances (green crosses in Figure 8) have $e_{\text {free }}$ that match the current four satellites, whereas no test particles match the current four satellites in the constant $\Delta t$ model. The probability of having test particles that are not affected by resonances is high (over 60\%) in the constant $Q$ model. Although the test particles that are not affected by resonances in the constant $Q$ model replicate the trend of Nix, Kerberos and Hydra in Figure 8, no preference for near resonant locations (in between 4:1 and 6:1 MMR) can be observed.
The different results obtained with constant $\Delta t$ and constant $Q$ may be due to the difference in tidal evolution timescale. As mentioned, the constant $Q$ model takes around 5-10 times longer to evolve. To test this, we increase the evolution timescale of the constant $\Delta t$ model by decreasing $\Delta t$ to half $(300 \mathrm{~s})$ or a quarter $(150 \mathrm{~s})$ of its original value, but keeping $A$ constant. Figure 9 shows a plot similar to Figure 8 , but including the results for $0.5 \Delta t$ (red squares) and $0.25 \Delta t$ (blue squares). We find that for a longer evolution timescale, test particles which are unaffected by resonances survive with slightly lower final $e_{\text {free }}$ (i.e., slightly closer to $e_{\text {free }}$ obtained for constant $Q$ ). This shows that increasing the tidal evolution timescale can decrease the final $e_{\text {free }}$ of the surviving test particles. However, we only tested a subset of 25 test particles and did not try even smaller $\Delta t$, and we cannot make a concrete conclusion on whether the constant $\Delta t$ model can also reproduce the eccentricities of the current satellites if $\Delta t$ is sufficiently small.

\subsection{Large $A$ and Initial $e_{C}=0$}

No test particles are ejected when initial $e_{\mathrm{C}}$ is set to 0 (see Table 3). The strength of the resonant terms depend on the eccentricities of both Charon and the test particle. Because $e_{\mathrm{C}}$ stays at zero and we have damped down $e_{\text {free }}$ of the test particles initially, the test particles are very unlikely to be affected by high-order resonances, even as low as 4:1 or 5:1. Therefore, the eccentricities of the test particles are not easily forced up to high values due to trapping in MMR with Charon, and their probability of survival is much higher than in the previous two cases.

In Figure 10, $98 \%$ of the test particles are in green, which indicates that most of the test particles are not affected by resonances. Their final $e_{\text {free }}$ are mostly within the range of $10^{-5}-10^{-4}$, which are at least an order of magnitude lower than the eccentricities of the current four small satellites. For the test particles not in green, they are either trapped in resonance or affected by resonances but not trapped. They all end near the 


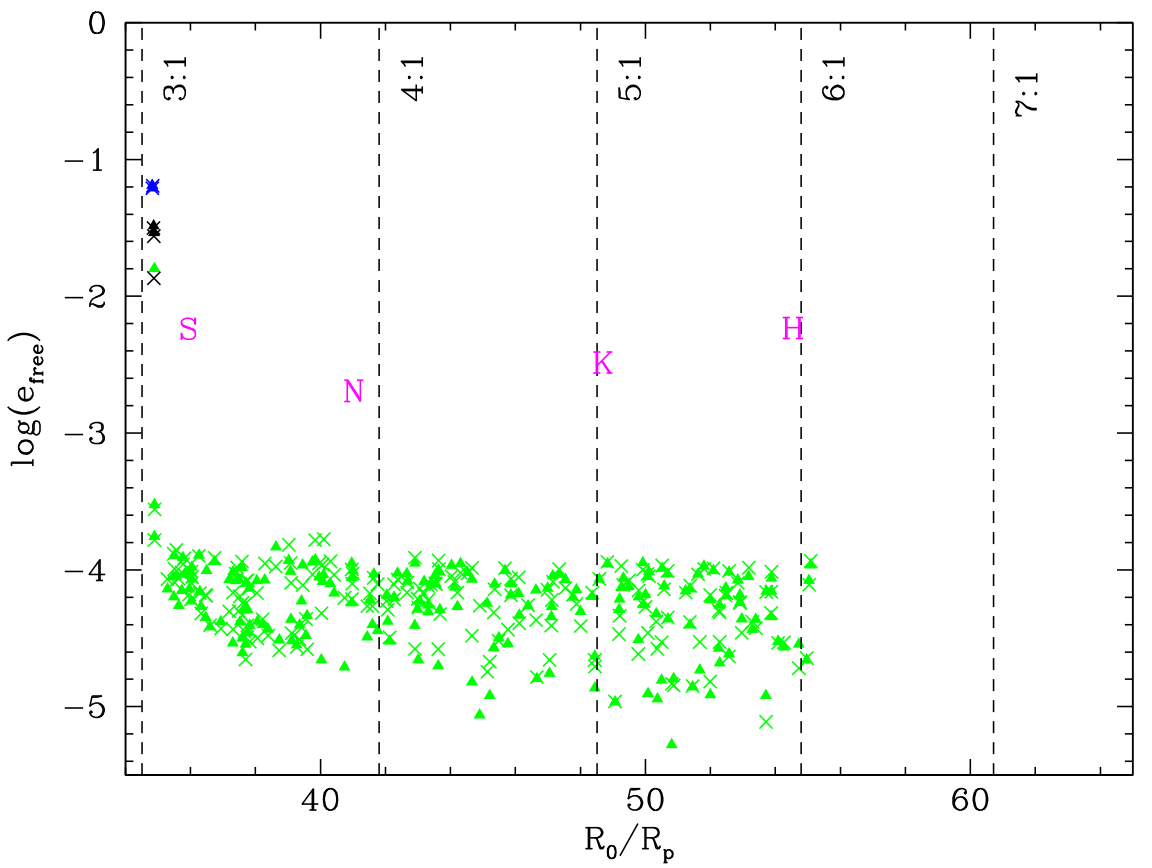

Figure 10. Same as Figures 7 and 8 , but for the large $A$ and initial $e_{\mathrm{C}}=0$ integrations.

3:1 MMR with Charon. Their $e_{\text {free }}$ are all within the range of $0.01-0.1$, which are more than twice the eccentricities of the current four small satellites. Compared to Styx, the test particles affected by resonances are located closer to the $3: 1$ MMR with Charon. Hence, we cannot find any test particles that match any one of the four small satellites in this case.

\section{Summary and Discussion}

We have investigated the early in situ formation scenario, which suggests that the four small satellites of Pluto formed in a debris disk near their current locations before the tidal evolution of Charon, by using $N$-body simulations to study the effects of the tidal evolution on the small satellites. The small satellites were treated as test particles that are initially collisionally damped to their coldest orbits, and the system was integrated in two different tidal models-constant $\Delta t$ and constant $Q$, with different relative rate of tidal dissipation in Charon and Pluto, $A$, and initial $e_{\mathrm{C}}$. The plausibility of the early in situ formation scenario was assessed by comparing the final $R_{0}$ and $e_{\text {free }}$ of the test particles with the actual values of the small satellites.

For large $A$ and initial $e_{\mathrm{C}}=0$, all of the test particles survive to the end. Most of the test particles are not affected by resonances and their final $e_{\text {free }}$ are at least an order of magnitude lower than those of the current satellites. Test particles that are affected by resonances are located closer to the 3:1 resonance than Styx, and their final $e_{\text {free }}$ are at least twice those of the current small satellites. Hence, we could not find any test particles with orbits similar to the current small satellites in this case. For small $A$ and initial $e_{\mathrm{C}}=0.2$, most of the test particles are ejected, and nothing can be found closer than the 5:1 resonance. Hence, this situation cannot explain the formation of Styx and Nix, which are now located closer than the 4:1 resonance. For large $A$ and initial $e_{\mathrm{C}}=0.2$, the results are different for the two tidal models. For constant $\Delta t$, we found that nothing matches the current satellites. For constant $Q$, we found that some test particles not affected by MMR survive with orbits similar to the orbits of the four small satellites. However, there is no preference for near resonance locations for these test particles. We also tested the relation between tidal evolution timescale and final $e_{\text {free }}$ for the test particles that are not affected by MMR by changing the $\Delta t$ value in the constant $\Delta t$ model. We discovered that increasing the tidal evolution timescale slightly decreases the final $e_{\text {free }}$ of the surviving test particles, but a more complete set of simulations is needed to determine whether the different results for the constant $\Delta t$ and constant $Q$ models are primarily due to the difference in the tidal evolution timescale.

To conclude, the only case with test particles that survive to the end of tidal evolution with similar orbits as the current four small satellites is constant $Q$ with large $A$ and initial $e_{\mathrm{C}}=0.2$. However, we still need to explain the near resonance locations of the small satellites for the early in situ formation scenario to work. As the probability of randomly forming the small satellites near MMR is low, there should be a reason for them to be near resonances, and a successful satellite formation model needs to account for this orbital feature.

We have assumed that the orbits of the small satellites are coplanar with that of Charon in our study. Quillen et al. (2017) have recently shown that the high obliquities of the small satellites (in particular, Styx and Nix) could be caused by commensurability between the MMR frequency and spin precession rate if the small satellites were captured into MMR involving inclination. It is unclear whether this mechanism could work in the context of the early in situ formation scenario, as their simulations show that the eccentricity of Nix is also excited to values much higher than the observed eccentricity, because the lowest-order $4: 1$ resonant angles containing the longitude of the ascending node $\Omega$ (which excite the inclination of Nix) also involve the longitude of periapse $\varpi$ (which excite the eccentricity of Nix). However, their simulations assume initial $e_{c}=0$. If $e_{c}$ is nonzero at the time of the resonance capture, it may be possible to keep the eccentricity of Nix small, as there are resonant 
angles involving the longitude of periapse of Charon $\varpi_{\mathrm{C}}$, but not $\varpi$.

We have neglected the masses of the small satellites in our study, and the masses of especially the more massive Nix and Hydra are an important factor in determining the long-term stability of the satellites. In particular, we may not need to explain the near resonance location of Kerberos, as it is located in the only stable region between Nix and Hydra (Pires dos Santos et al. 2011; Youdin et al. 2012). Youdin et al. (2012) found that the masses of Nix and Hydra should be smaller than $5 \times 10^{16} \mathrm{~kg}$ and $9 \times 10^{16} \mathrm{~kg}$, respectively, in order for Kerberos to be stable over the age of the solar system.

As mentioned, some of the other formation scenarios for the small satellites have been proven to be unsuccessful. For example, the collisional capture scenario, in which the small satellites were formed at their current locations from a collision between two planetesimals captured by Pluto-Charon after tidal evolution, was ruled out by Pires dos Santos et al. (2012), because they found that the timescale of temporary capture for a planetesimal that is massive enough to produce Nix and Hydra is much shorter than the timescale for another planetesimal to come in and collide with it. Their assumed masses for Nix and Hydra are based on the values reported by Tholen et al. (2008), which are $5.8 \times 10^{17} \mathrm{~kg}$ and $3.2 \times 10^{17} \mathrm{~kg}$, respectively. These are much higher than the nominal masses in the latest measurements by Showalter \& Hamilton (2015; see Table 1) and the mass constraints for Nix and Hydra in Youdin et al. (2012). If we study the collisional capture scenario by adopting the smaller masses from the latest measurement, a smaller difference in the timescales should be obtained.

Because the masses of Nix and Hydra are an important factor constraining the formation of the small satellites, we estimate their masses from the latest size measurements by the New Horizons spacecraft. Assuming that both are approximately ellipsoids, the size of Nix is $50 \times 35 \times 33 \mathrm{~km}( \pm 3 \mathrm{~km})$, and the size of Hydra is $65 \times 45 \times 25 \mathrm{~km}( \pm 10 \mathrm{~km}$; Weaver et al. 2016). If we assume that they are pure icy objects with density $\rho=1 \mathrm{~g} \mathrm{~cm}^{-3}$, the mass of Nix is $1.89 \times 10^{17}$ to $3.04 \times 10^{17} \mathrm{~kg}$, and the mass of Hydra is $1.21 \times 10^{17}$ to $6.05 \times 10^{17} \mathrm{~kg}( \pm 1 \sigma$ from size $)$. Even the lower masses of Nix and Hydra we just estimated are more than $1 \sigma$ above the upper limits measured by Showalter \& Hamilton (2015; see Table 1). Besides, the density we assumed is lower than the density of both Pluto $\left(\rho_{\mathrm{p}}=1.86 \mathrm{~g} \mathrm{~cm}^{-3}\right)$ and Charon $\left(\rho_{\mathrm{c}}=1.70 \mathrm{~g} \mathrm{~cm}^{-3}\right)$. The same timescale problem arises in the collisional capture scenario based on our estimated values, as our values are comparable to those of Tholen et al. (2008). On the other hand, our estimation for the masses is much larger than the upper limits for the masses of Nix and Hydra in order for Kerberos to stay at its current orbit for the age of the solar system, according to Youdin et al. (2012). We have to account for the stability of Kerberos, unless the Pluto satellite system was formed much later than the solar system. The high albedo of the small satellites (see Table 1) may be evidence for their late formation, but the densities of craters on Nix and Hydra suggest surface ages of at least 4 billion years (Weaver et al. 2016). A more precise measurement of the orbits of the small satellites by the New Horizons spacecraft and the Hubble Space Telescope may help us to accurately determine the masses of the satellites and hence understand the origin of the small satellites.

We thank Mark Showalter for providing the initial state vectors of Pluto's satellites from Showalter \& Hamilton. This work was supported by a postgraduate studentship at the University of Hong Kong (M.Y.W.) and Hong Kong RGC grant HKU 7030/11P (M.Y.W. and M.H.L.).

\section{ORCID iDs}

Man Hoi Lee (iD https://orcid.org/0000-0003-1930-5683

\section{References}

Bromley, B. C., \& Kenyon, S. J. 2015, ApJ, 809, 88

Brozović, M., Showalter, M. R., Jacobson, R. A., \& Buie, M. W. 2015, Icar, 246, 317

Canup, R. M. 2005, Sci, 307, 546

Cheng, W. H., Lee, M. H., \& Peale, S. J. 2014a, Icar, 233, 242

Cheng, W. H., Peale, S. J., \& Lee, M. H. 2014b, Icar, 241, 180

Christy, J. W., \& Harrington, R. S. 1978, AJ, 83, 1005

Holman, M. J., \& Wiegert, P. A. 1999, AJ, 117, 621

Kenyon, S. J., \& Bromley, B. C. 2014, AJ, 147, 8

Lee, M. H., \& Peale, S. J. 2002, ApJ, 567, 596

Lee, M. H., \& Peale, S. J. 2003, ApJ, 592, 1201

Lee, M. H., \& Peale, S. J. 2006, Icar, 184, 573

Leung, G. C. K., \& Lee, M. H. 2013, AJ, 763, 107

Levison, H. F., \& Duncan, M. J. 1994, Icar, 108, 18

Lithwick, Y., \& Wu, Y. 2008, arXiv:0802.2951

Mignard, F. 1980, M\&P, 23, 185

Murray, C. D., \& Dermott, S. F. 1999, Solar System Dynamics (Cambridge: Cambridge Univ. Press)

Pires dos Santos, P. M., Giuliatti Winter, S. M., \& Sfair, R. 2011, MNRAS, 410, 273

Pires dos Santos, P. M., Morbidelli, A., \& Nesvorný, D. 2012, CeMDA, 114,341

Quillen, A. C., Nichols-Fleming, F., Chen, Y.-Y., \& Noyelles, B. 2017, Icar, 293, 94

Showalter, M. R., \& Hamilton, D. P. 2015, Natur, 522, 45

Showalter, M. R., Hamilton, D. P., Stern, S. A., et al. 2011, in IAUC No. 9221, ed. D. W. E. Green (Cambridge: Cambridge Univ. Press), 1

Showalter, M. R., Weaver, H. A., Stern, S. A., et al. 2012, IAUC No. 9253 (Cambridge: Cambridge Univ. Press), 1

Smullen, R. A., \& Kratter, K. M. 2017, MNRAS, 466, 4480

Stern, S. A., Bagenal, F., Ennico, K., et al. 2015, Sci, 350, aad1815

Tholen, D. J., Buie, M. W., Grundy, W. M., \& Elliott, G. T. 2008, AJ, 135, 777

Walsh, K. J., \& Levison, H. F. 2015, AJ, 150, 11

Ward, W. R., \& Canup, R. M. 2006, Sci, 313, 1107

Weaver, H. A., Stern, S. A., Mutchler, M. J., et al. 2006, Natur, 439, 943

Weaver, H. A., Buie, M. W., Buratti, B. J., et al. 2016, Sci, 351, aae0030

Wisdom, J., \& Holman, M. 1991, AJ, 102, 1528

Woo, M. Y., \& Lee, M. H. 2018, AJ, submitted

Yoder, C. F., \& Peale, S. J. 1981, Icar, 47, 1

Youdin, A. N., Kratter, K. M., \& Kenyon, S. J. 2012, ApJ, 755, 17 